\title{
Head and Neck Part
}

National Cancer Institute

\section{Source}

National Cancer Institute. Head and Neck Part. NCI Thesaurus. Code C38617.

Any component associated with of the base of the skull and facial bones, sinuses, orbits, salivary glands, oral cavity, oropharynx, larynx, thyroid, facial and neck musculature and lymph nodes draining these areas. 